\title{
RF EXPERIMENTS ON Rb-RARE-GAS VAN DER WAALS MOLECULES
}

\author{
M. A. BOUCHIAT and L. C. POTTIER \\ Laboratoire de Spectroscopie Hertzienne de l'Ecole Normale Supérieure (*) \\ Faculté des Sciences de Paris, Paris, France
}

(Reçu le 5 octobre 1971)

\begin{abstract}
Résumé. - Nous avons détecté l'action d'un champ de radiofréquence sur les molécules $\mathrm{Rb}-\mathrm{Kr}$ et $\mathrm{Rb}$-Ar en utilisant des techniques de pompage optique. A de faibles pressions de gaz rare, on peut comparer cette expérience à celle de résonance magnétique de ces molécules. On obtient des informations significatives sur la variation de la force de couplage spin-orbite d'un état moléculaire de rotation-vibration à un autre. Les résultats expérimentaux préliminaires sont en très bon accord avec la théorie.

Abstract. - We have detected the action of a $r f$ field on Rb-Kr and Rb-Ar molecules by optical pumping techniques. At a low rare-gas pressure, the experiment can be compared with one of magnetic resonance performed on these molecules. It provides significant information about the dependence of the spin-orbit interaction strength on the rotation-vibrational state of the molecule. Preliminary experimental results are in excellent quantitative agreement with the theory.
\end{abstract}

In a mixture of krypton and rubidium vapor at room temperature $\left(\mathrm{Rb}\right.$ pressure $\approx 10^{-7}$ torr $)$, Van der Waals attractions give rise to the equilibrium $\mathrm{Rb}+\mathrm{Kr} \rightleftarrows \mathrm{Rb}-\mathrm{Kr}$. From the knowledge of the $\mathrm{Rb}-\mathrm{Kr}$ interatomic potential (welldepth $\varepsilon=10^{-2} \mathrm{eV}$; radius $r_{\mathrm{m}}=4.5 \AA$ ) it is possible to establish by simple statistical considerations [1] the law of mass action and to calculate the characteristic constant : the proportion of $\mathrm{Rb}-\mathrm{Kr}$ molecules at $300{ }^{\circ} \mathrm{K}$ is found to be $n_{\mathrm{Rb}-\mathrm{Kr}} / n_{\mathrm{Rb}}=5.35 \times 10^{-6} \mathrm{P}$ (where $P$ is the $\mathrm{Kr}$ pressure in torrs) ; at a $\mathrm{Kr}$ pressure of 1 torr, for instance, the partial pressure of $\mathrm{Rb}-\mathrm{Kr}$ is of the order of $5 \times 10^{-13}$ torr. Of course these equilibrium values result from continuous competition between generation and destruction of molecules. The molecular lifetime $\tau$ and generation rate $T_{\mathrm{f}}^{-1}$ (which have to satisfy the steady-state condition $n_{\mathrm{Rb}-\mathrm{Kr}} / \tau=n_{\mathrm{Rb}} / T_{\mathrm{f}}$ ) are respectively $\tau=5.7 \times 10^{-8} / P$ and $T_{\mathrm{f}}^{-1}=94 P^{2}$ ( $\tau$ in seconds, $T_{\mathrm{f}}^{-1}$ in $\mathrm{s}^{-1}, P$ in torrs).

Inside the molecule the spin $\mathbf{S}$ of the $\mathrm{Rb}$ atom is submitted to spin-orbit coupling $\gamma \mathbf{S}$.N with the orbital angular momentum $\mathbf{N}$ of the pair around its center of mass. Since $N$ is much greater than unity $(\bar{N}=40)$, $\mathbf{N}$ can, with good approximation, be treated as a classical vector: spin-orbit coupling can thus be regarded as the action of a «molecular field» [2] $\boldsymbol{\omega}_{1}=\gamma \mathbf{N} / \gamma_{\mathrm{s}}$. Since the discrete structure of $\mathbf{N}$ is too fine to be resolved in our experiments, $\omega_{1}$ can be considered to have a continuous distribution $\Pi\left(\omega_{1}\right)$.

(*) Associé au Centre National de la Recherche Scientifique, Paris, France.
We shall also assume (see ref. [1] for justification) that $\omega_{1}$ remains constant during the lifetime of the molecule; but of course it varies from one molecule to the other. Its r. m. s. magnitude is $9.6 \mathrm{G}$. In the case when the spins of the $\mathrm{Rb}$ atoms are oriented (e. g. by optical pumping), the random molecular field acts as a cause of relaxation, the rate of which we shall denote by $T_{\mathrm{S}}^{-1}$. Let $T_{1}^{*-1}$ be the relaxation rate due to all other processes, i. e. to wall collisions and usual gas collisions (without molecule formation). The total relaxation rate of the longitudinal orientation $\left\langle S_{\mathrm{z}}\right\rangle$ is thus given by

$$
T_{1}^{-1}=T_{S}^{-1}+T_{1}^{*-1} .
$$

From experimental measurements of $T_{1}^{-1}$ as a function of the external d. c. magnetic field $\omega_{0}$ the two rates $T_{\mathrm{S}}^{-1}$ and $T_{1}^{*-1}$ can easily be deduced separately, for $T_{\mathrm{S}}^{-1}$ strongly depends on $\omega_{0}$ in the range $0-200 \mathrm{G}$ whereas $T_{1}^{*-1}$ is field independent in the same range [1]. The molecular parameters (lifetime, molecular field, etc.) given above are deduced from the experimental study of $T_{\mathrm{S}}^{-1}$ versus $\omega_{0}$ and the krypton pressure $P$.

It would be interesting to obtain information about the shape of the distribution $\Pi\left(\omega_{1}\right)$ of the molecular field : since the distribution $\mathscr{T}(N)$ of $N$ is known theoretically [1], [3], a comparison of $\Pi\left(\omega_{1}\right)$ with $\mathscr{T}(N)$ would provide information about the dependence of the spin-orbit coupling constant $\gamma$ upon the molecular state $|v, N\rangle$. It appears, unfortunately, that the relaxation rate $T_{\mathrm{S}}^{-1}$ is fairly insensitive to the shape of the molecular field distribution, because all molecules with all values of $\omega_{1}$ participate simultaneously in the 
relaxation. To scan the distribution $\Pi\left(\omega_{1}\right)$ one should somehow select a narrow band of values of $\omega_{1}$. A possible method of doing so is to apply a $r f$ field $\omega_{1}^{\prime} \cos \omega^{\prime} \mathrm{t}$ so as to induce resonant spin-flips in the molecules for which the magnitude $\left|\boldsymbol{\omega}_{0}+\boldsymbol{\omega}_{1}\right|$ of the total field is equal to $\omega^{\prime}$ : this will cause $a$ change $\Delta\left(T_{\mathrm{S}}^{-1}\right)$ of the relaxation rate, and consequently a change $\left.\Delta<S_{\mathrm{z}}\right\rangle$ of the stationary longitudinal orientation of the vapor, which in principle can be detected experimentally. It is clear that the dependence of $\left.\Delta<S_{\mathrm{z}}\right\rangle$ upon $\omega^{\prime}$ will in some way reflect the distribution $\Pi\left(\omega_{1}\right)$.

The physically most interesting situations are those where the d. c. field $\omega_{0}$ is either much greater or much smaller than the average molecular field $\omega_{1}^{*}$. In the high-field case [4] $\left(\omega_{0} \gg \omega_{1}^{*}\right)$, the hamiltonian $\mathscr{H}=\omega_{0} S_{\mathbf{z}}+\omega_{1} \cdot \mathbf{S}_{1}$ can be written, to the first order : $\mathcal{H} \simeq\left(\omega_{0}+\omega_{1 \mathrm{z}}\right) S_{\mathrm{z}}$, so that a transverse $r f$ field at frequency $\omega^{\prime}$ will affect molecules for which

$$
\omega_{0}+\omega_{1 z}=\omega^{\prime}
$$

(inducing transitions $\Delta S_{\mathrm{z}}= \pm 1$ ) but should have no effect on free $\mathrm{Rb}$ atoms if $\omega^{\prime} \neq \omega_{0}$. In practice, since in the alkali atom the electronic spin $\mathbf{S}$ is coupled with the nuclear spin I, and because of the partial decoupling of $\mathbf{S}$ and $\mathbf{I}$ in the field $\omega_{0}$, atomic and molecular spectra are superimposed in the same frequency range, which makes the detection difficult. We shall limit ourselves here to the opposite case $\left(\omega_{0} \ll \omega_{1}^{*}\right)$ in which we have obtained significant experimental results. Since the total field $\omega_{0}+\omega_{1}$ can now take on any direction, it is advisable to apply the $r f$ field along $\omega_{0}$ so that it has no effect on free $\mathrm{Rb}$ atoms. Besides, the lifetime $\tau$ (i. e. the pressure) should be chosen so as to satisfy the following conditions :

$$
\omega^{\prime} \tau \gg 1, \quad\left(\tau^{-2}+\omega_{1}^{\prime 2}\right)^{1 / 2} \ll \dot{\delta} \omega_{1},
$$

where $\delta \omega_{1}$ is the width of the distribution of the molecular field $\omega_{1}$. The first condition means that the $r f$ field has time to perform several oscillations during the life of the molecule, so that it is actually felt as a $r f$ field ; the second one requires that the resonance line be sufficiently narrow for the method to be selective.

The change $\Delta\left(T_{\mathrm{S}}^{-1}\right) / T_{\mathrm{S}}^{-1}$ of the molecular relaxation rate under the effect of the $r f$ field can be calculated as follows : given a spin $\mathbf{S}$ obeying the equation of precession [5]

$$
\frac{\mathrm{d} \mathbf{S}}{\mathrm{d} t}=\omega \wedge \mathbf{S}
$$

and initially oriented along $\mathrm{Oz}\left(S_{\mathrm{z}}=1\right)$, submit it from instant $t_{0}$ to instant $t_{0}+T$ to a magnetic field [6] :

$$
\boldsymbol{\omega}=\omega_{1}+\omega_{1}^{\prime} \cos \omega^{\prime} t
$$

Let

$$
s=\left\langle S_{\mathrm{z}}\left(t_{0}+T\right)\right\rangle_{i_{0}, T, \omega_{1}}
$$

be the final value of $S_{\mathrm{z}}$, averaged over the random variables $t_{0}$ (instant of the molecule formation), $T$ (lifetime) and $\omega_{1}$ (molecular field); let $T_{\mathrm{f}}^{-1}$ be the average molecule formation rate. It can be shown that provided the condition $\tau \ll T_{\mathrm{f}}$ is satisfied, then the molecular relaxation rate $T_{\mathrm{S}}^{-1}$ is simply given by

$$
T_{\mathrm{S}}^{-1}=T_{\mathrm{f}}^{-1}(1-s),
$$

(i. e. the disorientation rate is equal to the loss of orientation at each interaction, times the frequency of the interactions).

The main difficulty is to solve equation (3) in the field (4). At sufficiently low pressures, when conditions (2) are satisfied, and if, furthermore, the frequency $\omega^{\prime}$ is close to the central value $\omega_{1}^{*}$ of the molecular field distribution, resonance effects will predominate. An approximate solution can then be derived by keeping only the resonant component of the $r f$ field (i. e. the direct circular component in a plane perpendicular to $\left.\boldsymbol{\omega}_{1}\right)$; in this approximation, the variation

$$
\Delta s=s\left(\omega_{1}^{\prime}\right)-s\left(\omega_{1}^{\prime}=0\right)
$$

of the quantity $s$ when the $r f$ field is turned on, is equal to :

$$
\Delta s=-\left\langle\frac{\left(\frac{\omega_{1}^{\prime}}{2} \sin \theta\right)^{2} \cos ^{2} \theta}{\frac{1}{\tau^{2}}+\left(\frac{\omega_{1}^{\prime}}{2} \sin \theta\right)^{2}+\left(\omega_{1}-\omega^{\prime}\right)^{2}}\right\rangle,
$$

where $\theta=\left(\mathrm{Oz}, \omega_{1}\right)$. Equation (8) means that for each value of $\omega_{1}$ and $\theta$, the relative variation $\Delta s / s$ (with $s=\cos ^{2} \theta$ ) of the final orientation $s$ under the effect of the direct circular $r f$ component (of modulus $\left.\left(\omega_{1}^{\prime} / 2\right) \sin \theta\right)$ is simply equal to the Rabi spin-flip probability. (Remembering that the $r f$ field is along $\mathrm{Oz}$, and that the molecular field is $\omega_{1}=\gamma \mathrm{N} / \gamma_{\mathrm{S}}$, one can see that the molecular transitions involved here are those for which $\Delta J_{z}=0, \Delta J= \pm 1$, where $\mathbf{J}=\mathbf{S}+\mathbf{N}$.) The ensuing variation $\Delta\left(T_{\mathrm{S}}^{-1}\right) / T_{\mathrm{S}}^{-1}$ is then, according to (6) :

$$
\frac{\Delta\left(T_{\mathrm{S}}^{-1}\right)}{T_{\mathrm{S}}^{-1}}=-\frac{\Delta s}{1-s} .
$$

In the low-pressure range $(P \leqslant 0.2$ torr) where this "resonance-approximation" is valid, $\Delta\left(T_{\mathrm{S}}^{-1}\right) / T_{\mathrm{S}}^{-1}$ is of the order of $+10^{-2}$ (the resonant $r f$ field at $\omega^{\prime} \# \omega_{1}^{*}=9.6 \mathrm{G}$ accelerates the relaxation).

At high pressures, on the contrary, when the condition $\omega \tau^{\prime} \ll 1$ is fulfilled, the $r f$ field has a very different effect : it acts as a d. c. field during the lifetime of the molecule. A reasonable approximation («quasistatic approximation ») is therefore to solve eq. (3) in the d. c. field $\boldsymbol{\omega}=\boldsymbol{\omega}_{1}+\boldsymbol{\omega}_{1}^{\prime} \cos \psi$, where $\psi$ is constant during the lifetime of the molecule but varies at random from one molecule to the other with a flat 
distribution between 0 and $2 \pi$. At pressures high enough for the conditions $\omega_{1}^{\prime} \tau \ll 1$ and $\omega_{1}^{*} \tau \ll 1$ to be fullfield, one finally obtains :

$$
\frac{\Delta\left(T_{\mathrm{S}}^{-1}\right)}{T_{\mathrm{S}}^{-1}}=-\frac{\omega_{1}^{\prime 2} \tau^{2}}{2} \text {. }
$$

Note that the $r f$ field (actually felt as a d. c. field) now slows down the relaxation. Besides, the value of $\Delta\left(T_{\mathrm{S}}^{-1}\right) / T_{\mathrm{S}}^{-1}$ now provides information about the lifetime $\tau$, but not about the molecular field distribution any longer.

It is clear that when the frequency $\omega^{\prime}$ becomes very large compared to $\omega_{1}^{*}$, the effect of the $r f$ field must be vanishingly small. An approximate analytical solution of eq. (3) and (4), valid when both conditions $\omega^{\prime} \tau \gg 1$ and $\omega^{\prime} \gg \omega_{1}^{*}$ (« high-frequency limit $\left.»\right)$ are fulfilled, shows that it decreases like $-\omega^{-2}$ at any pressure (the sign thus again corresponds to a slowing down of the relaxation). In intermediate cases where none of the above approximate treatments is valid, eq. (3) and (4) have to be solved numerically on a large computer.

Figure 1 shows in a qualitative way the behaviour of $\Delta\left(T_{\mathrm{S}}^{-1}\right) / T_{\mathrm{S}}^{-1}$ versus the frequency $\omega^{\prime}$ at various

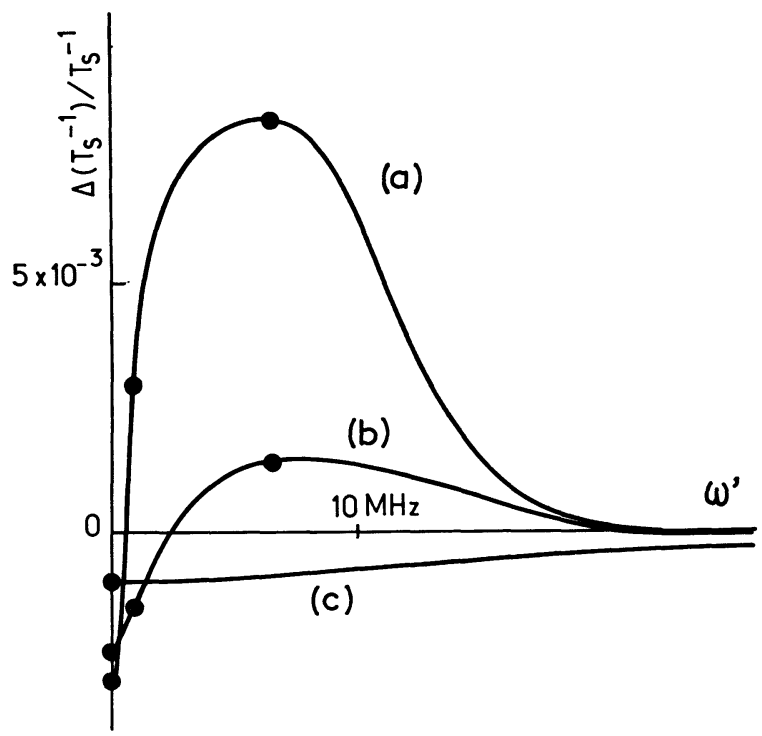

FIG. 1. - Effect of a $r f$ field (amplitude $\omega_{1}^{\prime}=0.85 \mathrm{G}$ ) on the relaxation rate $T_{\mathrm{S}}^{-1}$ of ${ }^{87} \mathrm{Rb}$ in $\mathrm{Kr}$ as a function of the $r f$ frequency, at three $\mathrm{Kr}$ pressures (curve a : 0.27 ; curbe b : 0.91 ; curve c : 4.75 torrs). The curves are qualitative, only the dots result from a quantitative calculation.

pressures. On the low-pressure curve (a), the three regions defined above (quasi-static, resonance and high-frequency regions) with their respective minus, plus and minus signs, are clearly visible. When the pressure is increased, the quasi-static region becomes larger and progressively " eats up » the resonance region (curve (b)) which at last disappears completely (curve (c)). Let us say again that the curves of figure 1 are purely qualitative (only the dots are the result of a correct calculation, performed under the assumption $\Pi\left(\omega_{1}\right)=\left(\gamma_{\mathrm{s}} / \gamma\right) \mathcal{T}(N)$.

Let us now describe the method used for the experimental detection. The effect of the $r f$ field is to modify the molecular relaxation rate $T_{\mathrm{S}}^{-1}$, and consequently the total rate $T_{1}^{-1}$ (see eq. (1)). The stationary value $\left\langle S_{\mathbf{z}}\right\rangle$ of the longitudinal orientation of the vapor results from the dynamical equilibrium between optical pumping and relaxation; more precisely,

$$
<S_{\mathrm{z}}>\propto \frac{1}{\left(1+T_{\mathrm{p}} / T_{1}\right)}
$$

where the "pumping-time » $T_{\mathrm{p}}$ characterizes the efficiency of the pumping process. $T_{\mathrm{p}}^{-1}$ is proportional to the intensity of the pumping beam [7]. According to (1) and (11), the change $\Delta\left(T_{\mathrm{S}}^{-1}\right) / T_{\mathrm{S}}^{-1}$ of the molecular relaxation rate due to the $r f$ field results in a change

$$
\frac{\Delta<S_{\mathrm{z}}>}{\left\langle S_{\mathrm{z}}\right\rangle}=-\frac{T_{1}}{T_{\mathrm{S}}} \cdot \frac{T_{\mathrm{p}} / T_{1}}{1+T_{\mathrm{p}} / T_{1}} \cdot \frac{\Delta\left(T_{\mathrm{S}}^{-1}\right)}{T_{\mathrm{S}}^{-1}}
$$

of the stationary orientation. This orientation change can be measured at several pumping-beam intensities and plotted as a function of the quantity $\left(T_{\mathrm{p}} / T_{1}\right) /\left(1+T_{\mathrm{p}} / T_{1}\right):$ according to (12), this yields a straight line of slope $-\left(T_{1} / T_{\mathrm{S}}\right) \cdot \Delta\left(T_{\mathrm{S}}^{-1}\right) / T_{\mathrm{S}}^{-1}$, whence $\Delta\left(T_{\mathrm{S}}^{-1}\right) / T_{\mathrm{S}}^{-1}$ is obtained. $\left(T_{1}\right.$ and $T_{\mathrm{S}}$ are known from separate relaxation measurements.)

The experiment has been performed on a set-up (Fig. 2) derived from the one of reference [8]: ${ }^{87} \mathrm{Rb}$

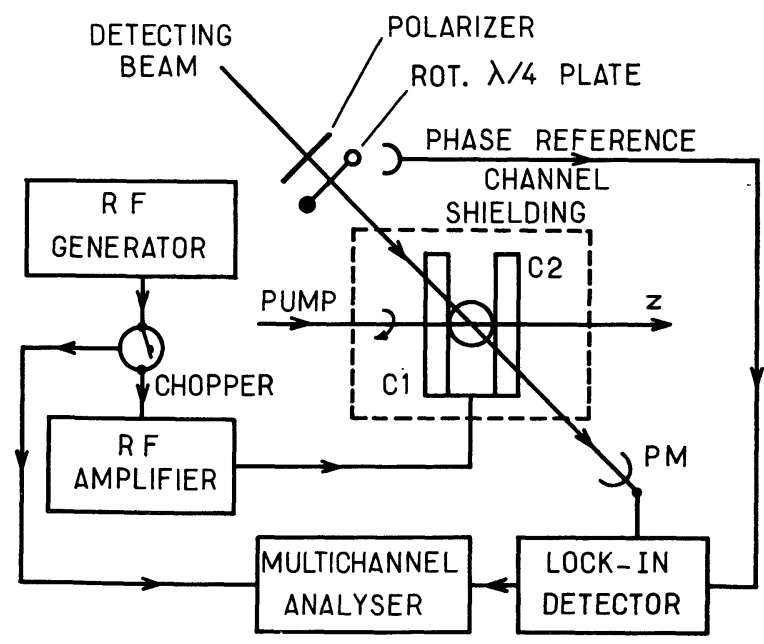

Fig. 2. - Experimental set-up ; C1, C2 : $r f$ coils ; d. c. field coils and details of the optics are not represented.

vapor, enclosed with $\mathrm{Kr}$ in a glass bulb, is optically oriented by a strong, circularly polarized pumping beam of resonant $\left(D_{1}\right)$ light. The orientation of the vapor is detected by means of a second resonant $\left(D_{2}\right)$ beam of low intensity. A quarterwave plate rotating behind a fixed polarizer modulates the polarization of 
TABLE I

Theoretical and experimental values of $\Delta\left(T_{\mathrm{S}}^{-1}\right) / T_{\mathrm{S}}^{-1}$
rf frequency $\omega^{\prime}=6.55 \mathrm{MHz} ;$ rf amplitude $\omega_{1}^{\prime}=0.85 \pm 0.042 \mathrm{G}$. All error bars are r. m. s. uncertainties

Pressure in torrs

$$
\text { Theor. I }
$$$$
\mathrm{Kr} \overline{0} .27
$$$$
\mathrm{Kr} 0.61
$$$$
\text { Kr } 0.91
$$$$
\text { Ar } 2.15
$$$$
\text { Ar } 4.85
$$

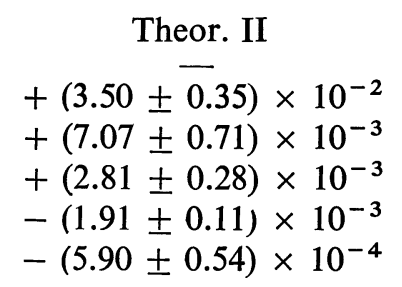

Theor. II

$+(3.50 \pm \overline{0.35}) \times 10^{-2}$

$+(7.07 \pm 0.71) \times 10^{-3}$

$+(2.81 \pm 0.28) \times 10^{-3}$

$-(1.91 \pm 0.11) \times 10^{-3}$

$-(5.90 \pm 0.54) \times 10^{-4}$

$$
\begin{gathered}
\text { Exp. } \\
+(7.81 \pm 0.66) \times 10^{-3} \\
+(3.42 \pm 0.20) \times 10^{-3} \\
+(1.26 \pm 0.08) \times 10^{-3} \\
-(1.45 \pm 0.24) \times 10^{-3} \\
-(4.01 \pm 1.19) \times 10^{-4}
\end{gathered}
$$

the detecting beam at a frequency of about $75 \mathrm{~Hz}$; the a. c. component of the transmitted intensity at the same frequency (detected by means of a lock-in detector) is proportional to the orientation of the vapor [9], [10]. The earth field is compensated (within a few milligauss) ; a d. c. field of about $0.2 \mathrm{G}$ is applied along the pumping direction $\mathrm{Oz}$. The $r f$ field, of amplitude $\omega_{1}^{\prime}=0.85 \mathrm{G}$ and frequency $\omega^{\prime}=9.35 \mathrm{G}$ (i. e. $6.55 \mathrm{MHz}$ ) \# $\omega_{1}^{*}$ is also directed along $\mathrm{Oz}$. A metal shielding prevents direct action of the $r f$ field on the electronics. Since the measurement has to be sensitive to fairly small orientation changes $\left.\left(\Delta<S_{\mathrm{z}}>/<S_{\mathrm{z}}\right\rangle \lesssim 10^{-3}\right)$, an averaging technique has been adopted : the $r f$ field is chopped at a frequency of $0.3 \mathrm{~Hz}$, and the ensuing chopped output signal of the lock-in detector is averaged in a multichannel analyzer. Relative orientation changes as small as $2 \times 10^{-4}$ can then be measured after one hour's averaging with an accuracy of about $10 \%$. The accuracy of the measurements is limited by the shot noise on the photocurrent (the intensity of the detecting beam must be kept low enough to play a negligible role in the relaxation).

$\mathrm{Up}$ to now we have measured $\Delta\left(T_{\mathrm{S}}^{-1}\right) / T_{\mathrm{S}}^{-1}$ at three krypton pressures, and also at two pressures of argon instead of krypton. Argon essentially behaves like krypton and most orders of magnitude are the same, except one major difference : the molecular field in $\mathrm{Rb}-\mathrm{Ar}$ is about 8 times smaller (r. m. s. value $\omega_{1}^{*} \approx 1.25 \mathrm{G}$ ) than in $\mathrm{Rb}-\mathrm{Kr}$. The situation is therefore quite different, since the frequency of our $r f$ field $\left(\omega^{\prime}=9.35 \mathrm{G}\right)$, resonant for $\mathrm{Rb}-\mathrm{Kr}$ molecules, is fully non-resonant for $\mathrm{Rb}-\mathrm{Ar}$ ones. Theoretical results (derived from the numerical treatment of eq. (3) and (4)) and experimental ones are presented in Table I. The second column (Theor. I) is obtained under the assumption $\gamma=$ constant, i. e. the dependence of the spin-orbit coupling constant upon the vibration-rotational state $|v, N\rangle$ is neglected: $\Pi\left(\omega_{1}\right)=\left(\gamma_{\mathrm{s}} / \gamma\right) \mathfrak{T}(N)$ [3]. The third column (Theor. II) corresponds to a molecular field distribution

$$
\Pi\left(\omega_{1}\right)=\delta\left(\omega_{1}-\omega_{1}^{*}\right),
$$

with

$$
\omega_{1}^{*} \approx \omega^{\prime}=9.35 \mathrm{G} .
$$

Since the $r f$ field amplitude $\omega_{1}^{\prime}=0.85 \pm 0.042 \mathrm{G}$ is known with finite accuracy, there is a corresponding uncertainty on the theoretical values. The inspection of Table I suggests the following comments :

1) in the case of $\mathrm{Kr}$ (low-pressure case, $\omega_{1}^{*} \tau \gg 1$ ) the results calculated with the "reasonable " distribution $\Pi\left(\omega_{1}\right) \propto \mathfrak{T}(N)$ agree with the experimental values, whereas those corresponding to the unrealistic shape $\Pi\left(\omega_{1}\right)=\delta\left(\omega_{1}-\omega_{1}^{*}\right)$ are too large by a factor of 2.5 to 4 : this shows that in the low-pressure region the method is actually sensitive to the shape of the molecular field distribution. (It is worth noticing that, on the contrary, the relaxation rates $T_{\mathrm{S}}^{-1}$ calculated with these two distributions are the same within $10 \%$.) In the opposite case, for Ar (high-pressure case, $\left.\omega_{1}^{*} \tau \ll 1\right)$, the distribution shape is irrelevant, in agreement with eq. (10).

2) it should be recalled here that the input parameters (lifetime $\tau$, molecular field $\omega_{1}^{*}$ ) of the theoretical calculation are simply the results of our previous relaxation measurements [1], [11], without any refinement. This establishes the consistency between the relaxation approach and the one described here.

3) the agreement obtained here at a frequency $\omega^{\prime} \# \omega_{1}^{*}$ (r. m. s. molecular frequency) essentially proves that the central region of our distribution $\Pi\left(\omega_{1}\right)$ is reasonable. It is not excluded, however, that future measurements performed at other frequencies (tails of the distribution) exhibit significant discrepancies between theory and experiment. This could provide interesting information about the dependence $\gamma(v, N)$ of the spin-orbit coupling constant upon the vibrational rotational state.

Acknowledgements. - The authors express their gratitude to Professor J. Brossel for constant help and encouragement during the course of the present work, and to Professor C. Bouchiat for useful comments. on the manuscript. 


\section{References and Footnotes}

(1) Bouchiat (C. C.), Bouchiat (M. A.) and Pottier (L. C.), Phys. Rev., 1969, 181, 144.

(2) Magnetic fields are expressed in pulsation units.

(3) The distribution $\mathbf{P}(N)$ is deduced from the Boltzmann distribution of the molecules in the vibration-rotational states $|v, N\rangle$, using the eigenenergies $E(v, N)$ computed from the $\mathrm{Rb}-\mathrm{Kr}$ potential.

(4) Bouchiat (C. C.) and Bouchiat (M. A.), Phys. Rev., 1970, A 4, 1274 .

(5) During a sticking collision $\left(\tau \approx 10^{-8} \mathrm{~s}\right)$ the nuclear spin I has time to couple with the electronic spin $\mathbf{S}$ (hf period $\approx 2 \times 10^{-10}$ s) so that $F$ is a good quantum number $(\mathbf{F}=\mathbf{I}+\mathbf{S})$ and $\mathbf{S}=g_{\mathbf{F}} \mathbf{F} ;$ moreover, the gyromagnetic factor $\gamma_{\mathbf{F}}=g_{\mathrm{F}} \gamma_{\mathbf{S}}$ has the same magnitude in both hfs levels $\left(g_{F}= \pm 1 /(2 I+1)\right)$. The nuclear spin I can therefore be formally ignored after simply dividing all Larmor frequencies by $2 I+1$.
(6) We now suppose $\omega_{0} \ll \omega_{1}$. so that $\omega_{0}$ can be neglected in the computations.

(7) In the case of an alkali atom, because of the nuclear spin, (11) actually holds only at low pumping-beam intensities.

(8) Bouchiat (M. A.) and Grossetête (F.), J. Physique, 1966, 27, 353.

(9) This is in principle equivalent to measuring $I\left(\sigma_{+}\right)-I\left(\sigma_{-}\right)$as described in reference [8]. However the present method gives in practice a better signal/noise ratio in the case of steady or slowly-varying phenomena (time-constants $>0.1 \mathrm{~s}$ ).

(10) The modulation ratio of the transmitted intensity is of the order of $5 \%$ for a total orientation of the vapor at $25{ }^{\circ} \mathrm{C}$.

(11) The value assumed for the amplitude of the $r f$ field $\left(\omega_{1}^{\prime}=0.85 \mathrm{G}\right)$ is also the result of a direct measurement. A discussion of the method employed is outside the scope of the present paper. 